\title{
Changes in dietary patterns when females engage in a weight management programme and their ability to meet Scientific Advisory Committee on Nutrition's fibre and sugar recommendations $\dagger$
}

\author{
Lauren Strathearn*, HK Kaçar and A Avery (1) \\ Division of Nutritional Sciences, School of Bioscience, University of Nottingham, Nottingham LE 12 5RD, UK
}

Submitted 30 January 2019: Final revision received 10 November 2019: Accepted 13 November 2019

\begin{abstract}
Objective: To investigate how dietary patterns (DP) change following engagement in a weight management programme. Using the DP identified, to study their relationship with weight loss achieved and ability to meet the Scientific Advisory Committee on Nutrition's 2015 fibre and sugar recommendations.

Design: Secondary analysis of FFQ data, which collected dietary information at two time points: retrospectively before (T0) and presently during (T1) Slimming World's programme, was analysed. Principal component analysis derived the DP present.

Setting: Online FFQ, hosted by Slimming World (SW) in the UK.

Participants: Female SW members ( $n$ 325; $17-68$ years old).

Results: At T0, two DP, 'plant based' and 'processed meat and sugar added by the consumer' were identified. At T1, three DP were identified, 'high meat', 'high fat salt and sugar (HFSS) products' and 'nuts and oils'. Participants with a diet low in 'HFSS products' lost significantly more weight ( $P$ for trend $=0.001)$, were more likely to consume breakfast $(P=0.021)$ and consumed less free sugar compared with high 'HFSS product' consumers. Those initially presenting with a low 'plant based' diet score lost more weight at T1 ( $P$ for trend $=0.046)$. With engagement, mean fibre intake increased from 24.3 to $32.4 \mathrm{~g} / \mathrm{d}(P<0.001)$ and free sugar intake decreased from 12.6 to $8.7 \%$ total dietary energy $(P<0.001)$.

Conclusions: An individual's DP before and with engagement in a weight management programme may indicate their weight loss success. Advising reduced HFSS product intake may aid initial weight loss. SW appears to promote dietary changes towards UK fibre and sugar recommendations.
\end{abstract}

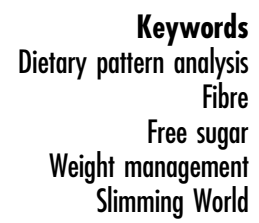

The obesity epidemic is becoming a widespread public health problem with $58 \%$ of women and $68 \%$ of men in 2015 being classed as overweight or obese in England ${ }^{(1)}$.

Recently, the evidence surrounding the effect of free sugars on weight gain has strengthened. From the available evidence, the Scientific Advisory Committee on Nutrition (SACN) concluded a higher sugar consumption increases energy intake in adults. Conversely, SACN suggested that a higher whole grain consumption may decrease total dietary energy intake. As a result, SACN recommended that free sugars should be reduced to a maximum $5 \%$ of total

$\dagger$ Research conducted at Division of Nutritional Sciences, School of Bioscience, University of Nottingham, Sutton Bonington Campus, Nottingham LE12 5RD, UK. dietary energy and suggested without energy substitution this could induce moderate weight loss. They further recommended increasing the dietary reference value for dietary fibre to $30 \mathrm{~g} / \mathrm{d}$, in adults 16 years and over ${ }^{(2)}$. The Eatwell plate was updated to (among other changes) reflect the SACN recommendations and this became the Eatwell guide $^{(3)}$.

For individuals who are overweight or obese, commercial weight management programmes are a popular, efficacious method of losing excess weight ${ }^{(4-6)}$, with the National Institute for Health and Care Excellence recommending these programmes as an option in first- and second-line treatment for individuals with overweight or obesity ${ }^{(7)}$. Slimming World (SW) is one such commercial weight 
management programme, including lifestyle and behaviour change strategies that patients can be referred to through primary care to receive free membership for a limited period, usually 12 weeks ${ }^{(4-6,8)}$.

SW's programme guides its members in making sustained healthy food and lifestyle choices through their Food Optimising plan ${ }^{(6)}$, encouraging members to alter their diet in line with the Eatwell guide ${ }^{(8)}$. Thus, it could be assumed by following SW's Food Optimising plan individuals will move towards achieving SACN's recommendations, reduce their energy consumption and lose weight ${ }^{(2,3)}$. However, this is yet to be investigated. Few studies have researched how dietary patterns (DP) change through engagement in a weight management programme in relation to weight $\operatorname{loss}^{(9-11)}$, and none have related this to SACN's recommendations for fibre and sugar ${ }^{(12)}$. Of these few studies, most involved online programmes which are known to have mixed effectiveness ${ }^{(13)}$ and none studied a UK population. DP are intrinsic to the country in which they are found; thus, the results from these studies cannot be generalised ${ }^{(14)}$.

The primary aim of this research is to determine the main DP present before and during engagement in SW's weight management programme. From this, identify how each DP correlates with weight loss and ability to meet SACN's dietary fibre and free sugar recommendations. It is theorised that a DP, in line with SW's Food Optimising plan, will correlate with greater weight loss, dietary fibre and a lower free sugar consumption. It is hypothesised participants' mean dietary fibre intake will significantly increase and free sugar intake will significantly decrease with engagement.

\section{Methods}

\section{Study design and population}

Data obtained from a lifestyle, dietary habits and Fe FFQ ${ }^{(15)}$, completed as part of a larger online survey investigating relationships between weight status and fatigue levels, were drawn upon for secondary analysis. The FFQ collected self-reported anthropometric, lifestyle and dietary information from female, non-pregnant, members of SW in the UK. The FFQ was made available on the SW members' website between 27 May 2016 and 10 June 2016. No reward was offered for participation.

In total, 825 members responded. Excluded from the analysis were 455 respondents with incomplete FFQ data and forty-five respondents who had been a member for less than 1 week. After these exclusions, 325 participants remained.

\section{The FFQ}

The FFQ has been validated, using Pearson's correlation coefficients, in a New Zealand population against a 4-d food record suggesting reasonable relative validity of 0.34-0.62 ( $P<0.001)$ for DP found, 'healthy' and 'sandwich and drinks', respectively. When repeated after 1 month, the correlation coefficient between FFQ for both DP was 0.76 $(P<0 \cdot 001)^{(15)}$. The FFQ was adapted for a UK population through changing food item names to their UK English equivalent. The FFQ comprised 133 food items with seven pre-coded frequency classifications ranging from 'never or less than once per month' to 'greater than four times per day'. In analysing responses, 1 month was taken as 4 weeks and all frequencies were converted into daily intakes in $g$. Participants reported their usual frequency of consumption of each food item at two time points simultaneously: retrospectively before joining SW (T0) and presently as a SW member (T1), see online supplementary material (S1).

Participants' intake of each food item in g per day was calculated through ${ }^{(15)}$ :

\section{daily frequency of consumption of each food item $x$ average portion size $(\mathrm{g})$ stated in the FFQ}

Estimations of participants' daily energy (kcal) and dietary fibre (Association of Analytical Chemists method) (g) consumption were calculated using daily intake of each food item (g) and nutritional information from McCance and Widdowson 'The composition of food' ${ }^{\text {(16) }}$. For food items not detailed in McCance and Widdowson, Nutritics Nutrition Analysis Software ${ }^{(17)}$ was used. Public Health England's definition of free sugars is used to calculate free sugar content of food items: 'all added sugars in any form; all sugars naturally present in fruit and vegetable juices, purees and pastes and similar products in which the structure has been broken down; all sugars in drinks (except for dairy-based drinks); and lactose and galactose added as ingredients. The sugars naturally present in milk and dairy products, fresh and most types of processed fruit and vegetables and in cereal grains, nuts and seeds are excluded from the definition, ${ }^{(18)}$. Free sugar (\% total dietary energy) content of food items was obtained from a published database detailing the amount of sugar added during manufacture of commonly consumed foods ${ }^{(19)}$; for fruit and vegetable juices and drinks (excluding dairy milk), the total sugar content of the food item was classed as a free sugar. Prior to analysis, the 133 food items were collapsed into thirty food groups based upon a previously published DP analysis ${ }^{(20)}$, online supplementary material (S2).

It is acknowledged that fibre intake is commonly overestimated in FFQ, mainly due to bias responses in fruit and vegetable consumption, with a higher discrepancy with those on a weight reducing diet. In the current study, dietary fibre intake was adjusted using a weighting factor developed to correct overestimates of fibre in UK women aged $35-69^{(21)}$.

Participants self-reported their body weight before and with engagement in SW in any unit. All weights were converted to $\mathrm{kg}$ for analysis. The physical activity levels were collected through participants reporting the number 
of days they had 'walked', conducted 'vigorous' and 'moderate' physical activity for more than $10 \mathrm{~min}$ at a time. Participants reported the time spent doing each of the intensity levels to the nearest minute before SW and with engagement in SW.

\section{Statistical analysis}

Principal component analysis (PCA) is a statistical method of analysing DP. PCA is a variable reduction procedure using correlations between food group consumption to generate a smaller number of uncorrelated DP, termed principal components. PCA captures the diet in its entirety, in a way that traditional methods often cannot ${ }^{(10,22,23)}$. The identified DP explain the majority of the variance present in the dataset ${ }^{(24)}$. PCA can discern the relationships between DP and participants' characteristics, weight loss and ability to meet dietary guidelines ${ }^{(22)}$.

The analysis of DP was conducted separately for T0 and T1 data. Prior to analysis, participants' daily intake (g/d) of each food group was energy adjusted, so that it acts as a measure of diet composition ${ }^{(25)}$ :

$$
\frac{\text { daily intake of food group }(\mathrm{g} / \mathrm{d})}{\text { total daily energy intake }(\mathrm{kcal})} \times 1000
$$

and then transformed using a $\log _{10}($ variable +1$)$ transformation to achieve a normal distribution for entry into PCA ${ }^{(24)}$.

To ascertain the suitability of the data for PCA to return reliable and accurate DP, and adequate sample size, the Kaiser-Meyer-Olkin measurement of adequacy and Bartlett's test of sphericity were determined ${ }^{(26)}$. The energyadjusted and transformed frequency values were entered into PCA in $\mathrm{IBM}^{\circledR}$ SPSS $^{\circledR}$ statistics, version $22^{(27)}$. The components (DP) were rotated using the varimax (orthogonal) rotation to enable interpretability of the food group loadings on the components. Food groups with loadings $>|0.3|$ were retained as part of the component solution

The Anderson-Rubin method was used to calculate a factor score detailing how the participants' dietary intake matched each $\mathrm{DP}^{(26)}$. Using the factor score, participants were grouped into quartiles (Q) of agreement for each DP. Quartile of factor score was compared with participants' characteristics and nutrient intake. All $P$ values are two-sided. To determine $P$ for trend between quartiles of factor score and participants' characteristics, the JonckheereTerpstra test was used. McNemar's test was used to determine if the proportion of participants meeting SACN's recommendations between T0 and T1 is statistically different. The chi square test was used to detect if the proportion of participants consuming breakfast always, sometimes or never, between quartiles of factor score is significantly different. Paired $t$ tests determined significance of change in dietary intake of the food groups between T0 and T1, and Pearson's correlation was used to determine the relationship between dietary fibre and free sugar consumption on weight loss achieved and to assess if exercise frequency at T1 influenced weight loss.

\section{Results}

\section{Characteristics of the participants}

Of the 325 participants eligible for entry into PCA, the mean age on completion of the questionnaire was 42.6 years (range 17-68), mean starting weight was $96 \cdot 0$ (range $59 \cdot 2-165 \cdot 1$; SD 20.6) $\mathrm{kg}$ and BMI was $35 \cdot 1$ (range $22.5-60 \cdot 2 ; \mathrm{SD} 7.2$ ) $\mathrm{kg} / \mathrm{m}^{2}$. Of the participants, $77 \%$ were in current employment, full or part-time and $8.7 \%$ were current smokers. Median length of SW membership of the 325 participants eligible for PCA was 3.3 (range 1-39; SD 3.2) weeks. At T1, 87.5\% of participants self-reported a weight loss, with a mean change in body weight of $-3 \cdot 2$ (SD 3.7) $\mathrm{kg}$ and mean change in percentage body weight of -3.3 (SD 3.2 ) \%. Participants had a mean body weight change per week of engagement of $-1 \cdot 1$ (SD 0.9) kg/week. No significant difference in age was apparent between completers and non-completers, not included in the analysis $(P=0 \cdot 199)$.

\section{Dietary patterns}

At T0, two DP and, at T1, three DP were identified and retained from PCA. Table 1 depicts the meaningful food group loadings on each DP. The Kaiser-Meyer-Olkin measure of sampling adequacy was 0.717 and 0.643 for $\mathrm{T} 0$ and $\mathrm{T} 1$, respectively, $>0.5$ is acceptable. The Bartlett's test of sphericity was below the acceptable level at $<0.001$, at both time points. At T0, component 1 was termed 'plant based'. Food groups with meaningful loadings (associations), $>|0 \cdot 3|$, on this component were legumes, fresh fruit, vegetables, whole grains, eggs, tea and coffee, soup, yoghurt and fish. The second component was termed 'processed meat and sugar added by the consumer' with processed meat and table sugar being strongly associated with this component. These two components explained $17 \cdot 6 \%$ of the variance, between participants' FFQ responses.

At T1, PCA revealed a different set of DP. The first DP identified was named 'high meat'. Processed meat, red meat and poultry had positive associations, whilst alternative milks were negatively associated with this component. The second component identified was named 'high fat, salt and sugar (HFSS) products'. Confectionery, crisps, cakes and biscuits, spreads and non-alcoholic drinks had strong associations with this pattern. The final meaningful DP at T1 was named 'nuts and oils', characterised by positive associations with dried fruit, alternative milks, nuts and seeds and oils, whilst potatoes negatively associated with the DP. These three components explained $21.0 \%$ of the variance between participants' FFQ responses.

The changes in DP between T0 and T1 reflect from changes in energy-adjusted food group consumption. The mean percentage change in energy-adjusted food group consumption of the participants is displayed in Fig. 1. At T1, the participants were consuming significantly 
Table 1 Meaningful rotated component loadings for retained dietary patterns before joining Slimming World (T0) and after joining Slimming World (T1)*

\begin{tabular}{|c|c|c|c|c|c|}
\hline \multirow[b]{2}{*}{ Food items } & \multicolumn{2}{|c|}{ TO } & \multicolumn{3}{|c|}{ T1 } \\
\hline & Component 1 & Component 2 & Component 1 & Component 2 & Component 3 \\
\hline Number of subjects $(n)$ & 325 & 325 & 325 & 325 & 325 \\
\hline Legumes & 0.708 & - & - & - & - \\
\hline Vegetables & 0.699 & - & - & - & - \\
\hline Whole grains & 0.644 & - & - & - & - \\
\hline Fresh fruit & 0.624 & -0.302 & - & - & - \\
\hline Eggs & 0.519 & - & - & - & - \\
\hline Tea and coffee & 0.430 & - & - & - & - \\
\hline Soup & 0.344 & - & - & - & - \\
\hline Fish & 0.358 & - & - & - & - \\
\hline Yoghurt & 0.317 & - & - & - & - \\
\hline Processed meat & - & 0.905 & 0.701 & - & - \\
\hline Table sugar† & - & 0.903 & - & - & - \\
\hline Red meat & - & - & 0.791 & - & - \\
\hline Poultry & - & - & 0.748 & - & - \\
\hline Confectionery & - & - & - & 0.708 & - \\
\hline Crisps & - & - & - & 0.709 & - \\
\hline Cakes and biscuits & - & - & - & 0.657 & - \\
\hline Non-alcoholic drinks & - & - & - & 0.358 & - \\
\hline Spreads & - & - & - & 0.446 & - \\
\hline Alternative milks & - & - & -0.377 & - & 0.392 \\
\hline Dried fruit & - & - & - & - & 0.612 \\
\hline Nuts and seeds & - & - & - & - & 0.629 \\
\hline Potato & - & - & - & - & -0.489 \\
\hline Oils & - & - & - & - & 0.597 \\
\hline Cows' milk & - & - & - & - & - \\
\hline Refined grains & - & - & - & - & - \\
\hline Alcohol & - & - & - & - & - \\
\hline Fruit and vegetable juice & - & - & - & - & - \\
\hline Breakfast cereal & - & - & - & - & - \\
\hline Cream-based products & - & - & - & - & - \\
\hline
\end{tabular}

*At T0, component 1 was termed 'plant based' and component 2 was termed 'processed meat and sugar added by the consumer'. At T1, component 1 was termed 'high meat', component 2 was termed 'high fat, salt and sugar products' and component 3 was termed 'nuts and oils'. †Table sugar added to foods and drink by the participant.

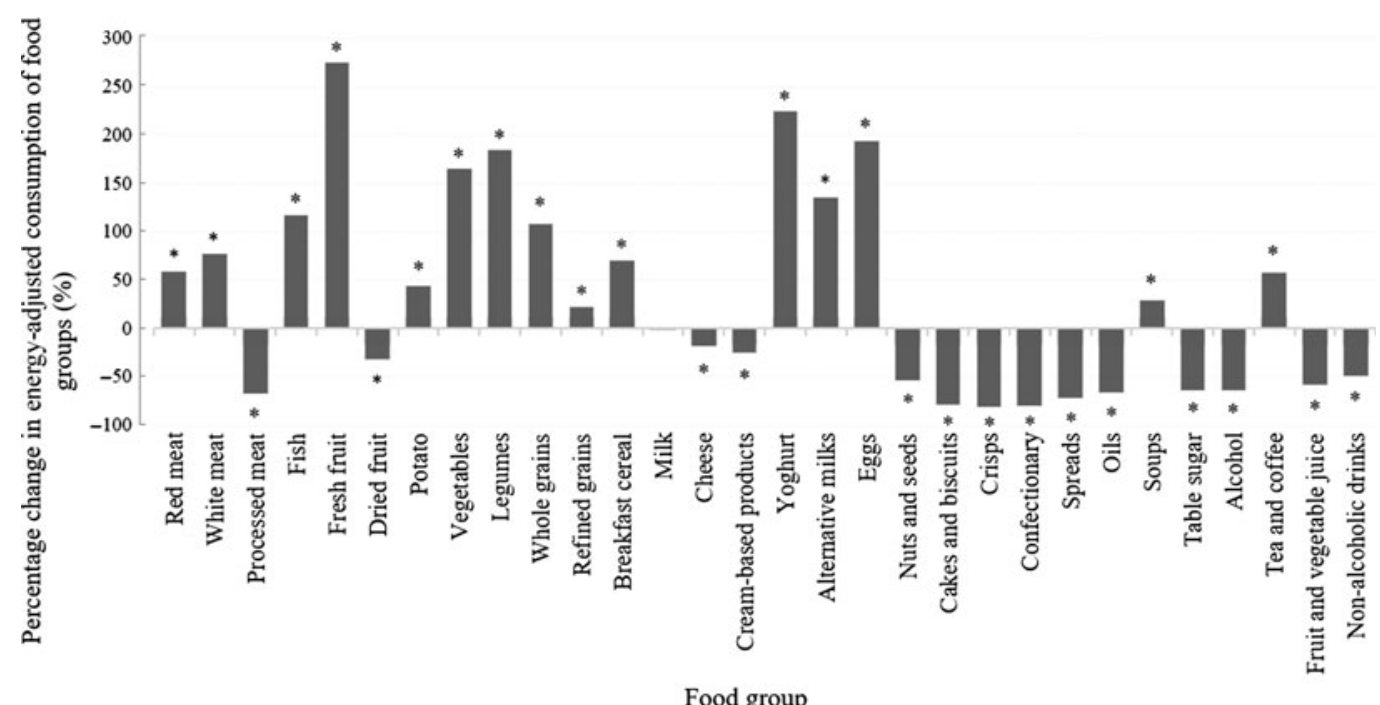

Fig. 1 Mean percentage change in energy-adjusted consumption of food groups between before Slimming World (T0) and during Slimming World membership (T1). Significance was calculated through a paired $t$ test. The percentage change in energy-adjusted consumption of all food groups was significant at the $P<0.05$ level (indicated by *) except for milk 
more fish, fresh fruit, vegetables and whole grains, whilst consuming significantly less processed meat, cheese, confectionery, crisps and cakes and biscuits.

\section{Participants' characteristics by dietary pattern for each component}

For each DP, participants were grouped into quartile of agreement to that DP. Participants were grouped such that Q1 contains participants with a very low score, diet did not reflect the DP, whilst Q4 contains participants with a very high score, dietary agreement, for the DP. Quartiles of factor score at T0 and T1 were compared with participants' characteristics separately. At T0, those with a high 'plant based' pattern score had significantly lower energy (kcal/d) intakes (mean Q4 $10259 \mathrm{KJ} / \mathrm{d}(2452 \mathrm{kcal} / \mathrm{d}), \mathrm{Q} 1$ $15108 \mathrm{KJ} / \mathrm{d}(3611 \mathrm{kcal} / \mathrm{d}) ; P$ for trend < $0 \cdot 001)$, were older (mean Q4 44.0 years, Q1 39.4 years; $P$ for trend $=0.003$ ), had a lower starting BMI (mean Q4 $32.9 \mathrm{~kg} / \mathrm{m}^{2}$, Q1 $36.8 \mathrm{~kg} / \mathrm{m}^{2} ; P$ for trend $\left.=0.001\right)$ and were more likely to consume vitamin and mineral supplements (mean Q4 $51.3 \%$ of participants consumed supplements, Q1 25.9\% consumed supplements; $P$ for trend $=0.001$ ). Those in the highest quartile of 'processed meat and sugar added by the consumer' pattern were significantly younger (mean Q4 39.4 years, Q1 44.3 years; $P$ for trend $=0.006$ ), exercised on a more frequent basis (mean Q4 6.1 frequency/ week, Q1 4.5 frequency/week; $P$ for trend $=0.005)$ and more likely to be a current smoker $(P$ for trend $=0.002$; Table 2).

At T1, participants in the highest quartile of the 'high meat' pattern consumed significantly less energy (mean Q4 $6899 \mathrm{KJ} / \mathrm{d}(1649 \mathrm{kcal} / \mathrm{d})$, Q1 $9878 \mathrm{KJ} / \mathrm{d}(2361 \mathrm{kcal} / \mathrm{d}) ; P$ for trend $<0.001)$. Those in the highest quartile of 'HFSS products' pattern were significantly younger (mean Q4 37.5 years, Q1 46.0 years; $P$ for trend $<0.001$ ) and had higher energy intakes (mean Q4 $9113 \mathrm{KJ} / \mathrm{d}(2178 \mathrm{kcal} / \mathrm{d})$, Q1 $7251 \mathrm{~kJ} / \mathrm{d}(1733 \mathrm{kcal} / \mathrm{d})$; $P$ for trend <0.001). A high 'nuts and oils' factor score correlated with higher energy intakes (mean Q4 $9498 \mathrm{KJ} / \mathrm{d}(2270 \mathrm{kcal} / \mathrm{d})$, Q1 $7058 \mathrm{KJ} / \mathrm{d}$ $(1687 \mathrm{kcal} / \mathrm{d}) ; P$ for trend $=0.004)$, a higher frequency of exercise (mean Q4 9.1 frequency/week, Q1 $7 \cdot 3$ frequency/week; $P$ for trend $=0.013$ ) and older age (mean Q4 44.5 years, Q1 39.9 years; $P$ for tend $=0.007$ ). Those aligning most strongly with a 'nuts and oils' DP had been a SW member for a significantly shorter period ( $P$ for trend =0.026; Table 3).

Of those initially following a 'plant based' pattern at T0, $34.9 \%$ changed their diets to most strongly align with a 'high meat' pattern, $37 \cdot 2 \%$ aligned with a 'HFSS products' pattern and $27.9 \%$ with a 'nuts and oils' pattern at T1. Of those initially following a 'processed meat and sugar added by the consumer' pattern at T0, $41.4 \%$ most closely aligned with a 'high meat' pattern, $21.4 \%$ with a 'HFSS products' pattern and $37 \cdot 1 \%$ with a 'nuts and oils' pattern at T1 (data not shown).

\section{Dietary patterns and weight loss}

Quartile of DP score, at T0, was compared with percentage weight change between T0 and T1. Participants with the lowest 'plant based' pattern score lost a significantly greater percentage body weight than those with the highest 'plant based' pattern score (mean Q1 -3.6\%, Q4 -2.8\%; $P$ for trend $=0.046$ ). The relationship between quartile of 'processed meat and sugar added by the consumer' pattern and weight loss was non-significant ( $P=0 \cdot 249$; Fig. 2$)$. The relationship between exercise frequency at T1 and weight loss achieved was non-significant $(r=0.037 ; P=0.505)$, and the Pearson's correlation between age and weight loss achieved was $r=-0.11$ indicating a weak negative correlation (data not shown).

At T1, those who aligned least strongly with a 'HFSS product' pattern lost a significantly greater percentage body weight (mean Q1 -4.1\%, Q4 -2.2\%; P for trend = 0.001). These individuals were significantly more likely to be consuming breakfast compared with those who strongly aligned with a 'HFSS products' pattern $(P=0 \cdot 021)$. No other significant relationships between the other DP at T1 and weight loss were observed.

\section{Fibre and free sugar consumption}

Before joining SW, mean dietary fibre intake was $24.3(\mathrm{SD} 19.8) \mathrm{g} / \mathrm{d}$ and mean free sugar consumption was 12.6 (SD 4.6 ) \% of total dietary energy with 23.0 and $5.4 \%$ of participants meeting the respective SACN recommendations. At T1, mean dietary fibre had significantly increased to $32.4(\mathrm{SD} 16.5) \mathrm{g} / \mathrm{d}(P<0.001 ; \mathrm{CI} 6.4,10 \cdot 0)$ and free sugar intake had significantly decreased to 8.7 (SD 3.6) \% $(P<0.001$; CI $-4.4,-3.4)$ of total dietary energy. At T1, 45.8 and $11.7 \%$ of participants met the respective SACN recommendations. No statistically significant relationship between dietary fibre or free sugar intake and weight loss was observed $(r=0.066 ; P=0.237$ and $r=-0 \cdot 107 ; P=0.056$, respectively).

Free sugar intake (\% total dietary energy) was compared with energy consumption at T0 and T1. At T0, there was a significant positive correlation between free sugar and energy intake $(r=0.264 ; P<0.001)$. At T1, there was no significant correlation between free sugar consumption and energy intake $(r=0.06 ; P=0.284)$.

Following a 'plant based' DP at T0 correlated with a higher dietary fibre consumption, as did a DP low in 'processed meat and sugar added by the consumer'. Those in the highest quartile of 'plant based' DP consumed significantly less free sugar than those in the lowest quartile (mean Q4 10.4\% total dietary energy, Q1 14.6\% total dietary energy; $P$ for trend $<0.001$; Table 2 ). At T1, those aligning most strongly with the 'high meat' DP had a lower intake of dietary fibre and free sugar compared with those in the lowest quartile ( $P$ for trend $<0.001$ and $<0.019$, respectively). Free sugar consumption positively correlated with agreement with the 'HFSS products' pattern ( $P$ for trend $<0.001$; Table 3). 
Table 2 Mean characteristics of participants by quartile (Q) of factor score for components identified before engagement in Slimming World (T0)

\begin{tabular}{|c|c|c|c|c|c|c|c|c|}
\hline \multirow[b]{3}{*}{ Characteristics } & \multicolumn{4}{|c|}{ Component 1} & \multicolumn{4}{|c|}{ Component 2} \\
\hline & \multicolumn{4}{|c|}{ Plant based } & \multicolumn{4}{|c|}{$\begin{array}{l}\text { Processed meat and sugar } \\
\text { added by the consumer }\end{array}$} \\
\hline & Q1 & Q2 & Q3 & Q4 & Q1 & Q2 & Q3 & Q4 \\
\hline Number of subjects $(n)$ & 81 & 82 & 81 & 81 & 81 & 82 & 81 & 81 \\
\hline Energy $(\mathrm{KJ} / \mathrm{d}(\mathrm{kcal} / \mathrm{d}))$ & $15108(3611)$ & $12121(2897)$ & $11770(2813)$ & $10259(2452)^{*}$ & $12401(2964)$ & $11067(2645)$ & 12707 (3037) & $13071(3124)$ \\
\hline Age on questionnaire completion (years) & 39.4 & $42 \cdot 1$ & $42 \cdot 7$ & $44.0 \dagger$ & $44 \cdot 3$ & $44 \cdot 1$ & $41 \cdot 7$ & $39.4 \dagger$ \\
\hline Weight BSW (kg) & $100 \cdot 0$ & $98 \cdot 3$ & $95 \cdot 7$ & $89.7 \dagger$ & $94 \cdot 1$ & 93.6 & 99.8 & $96 \cdot 2$ \\
\hline BMI BSW $\left(\mathrm{kg} / \mathrm{m}^{2}\right)$ & $36 \cdot 8$ & 35.9 & 34.7 & $32.9+$ & 34.9 & $34 \cdot 3$ & $35 \cdot 8$ & $35 \cdot \overline{3}$ \\
\hline Percentage weight loss (\%) & $-3 \cdot 6$ & $-3 \cdot 3$ & -3.4 & $-2 \cdot 8 \S$ & $-3 \cdot 2$ & $-3 \cdot 4$ & $-3 \cdot 3$ & $-3 \cdot 1$ \\
\hline Current smokers (\%) & $16 \cdot 0$ & 2.5 & 8.6 & 7.5 & 3.7 & $5 \cdot 0$ & 8.6 & $17 \cdot 3 \dagger$ \\
\hline Exercise (frequency /week) & 4.5 & 4.9 & 5.4 & $5 \cdot 9$ & 4.5 & 4.5 & $5 \cdot 6$ & $6 \cdot 1 \dagger$ \\
\hline Employed (\%) & $79 \cdot 0$ & $76 \cdot 3$ & $76 \cdot 5$ & $76 \cdot 3$ & $76 \cdot 5$ & $77 \cdot 2$ & 81.5 & $72 \cdot 8$ \\
\hline Vitamin and mineral supplement use (\%) & $25 \cdot 9$ & $35 \cdot 8$ & $38 \cdot 3$ & $51 \cdot 3 \dagger$ & 43.2 & $36 \cdot 3$ & $42 \cdot 0$ & $26 \cdot 6$ \\
\hline \multicolumn{9}{|l|}{ Breakfast (\%) } \\
\hline Always & $42 \cdot 0$ & $50 \cdot 0$ & $60 \cdot 3$ & $56 \cdot 3$ & $55 \cdot 6$ & $61 \cdot 0$ & $60 \cdot 6$ & 47.5 \\
\hline Sometimes & $24 \cdot 7$ & $23 \cdot 8$ & $23 \cdot 1$ & $22 \cdot 0$ & $26 \cdot 3$ & $14 \cdot 3$ & $24 \cdot 0$ & 22.5 \\
\hline Never & $30 \cdot 1$ & $33 \cdot 3$ & 13.6 & $14 \cdot 6$ & $19 \cdot 7$ & $22 \cdot 5$ & $20 \cdot 2$ & $29 \cdot 2$ \\
\hline Fibre $(g / d)$ & $22 \cdot 5$ & 22.5 & 24.5 & $27 \cdot 6 \dagger$ & $26 \cdot 8$ & $25 \cdot 5$ & $24 \cdot 6$ & $20 \cdot 2 \dagger$ \\
\hline Free sugar (\% total kcal) & $14 \cdot 6$ & $13 \cdot 1$ & $12 \cdot 2$ & $10 \cdot 5^{\star}$ & $12 \cdot 7$ & $12 \cdot 7$ & $13 \cdot 2$ & 11.8 \\
\hline
\end{tabular}

BSW, before Slimming World.

${ }^{*} P$-values $<0.05$ were considered as significant. Exercise expressed as total frequency of vigorous and moderate physical activity and walking events lasting for at least $10 \mathrm{~min} / \mathrm{event}$ per week. $\dagger P$ for trend $<0.001$ calculated using Jonckheere-Terpstra test.

$\ddagger P$ for trend $0.001<P<0.025$ calculated using Jonckheere-Terpstra test.

$\S P$ for trend $0.025<P<0.05$ calculated using Jonckheere-Terpstra test.

Otherwise no significant $P$ for trend. 
Table 3 Mean characteristics of participants by quartile of factor score for dietary patterns identified with engagement in Slimming World (T1)

\begin{tabular}{|c|c|c|c|c|c|c|c|c|c|c|c|c|}
\hline \multirow[b]{3}{*}{ Characteristics } & \multicolumn{4}{|c|}{ Component 1} & \multicolumn{4}{|c|}{ Component 2} & \multicolumn{4}{|c|}{ Component 3} \\
\hline & \multicolumn{4}{|c|}{ High meat } & \multicolumn{4}{|c|}{ HFSS products } & \multicolumn{4}{|c|}{ Nuts and oils } \\
\hline & Q1 & Q2 & Q3 & Q4 & Q1 & Q2 & Q3 & Q4 & Q1 & Q2 & Q3 & Q4 \\
\hline Number of subjects $(n)$ & 81 & 82 & 81 & 81 & 81 & 82 & 81 & 81 & 81 & 81 & 82 & 81 \\
\hline $\begin{array}{l}\text { Length of membership } \\
\text { (weeks) }\end{array}$ & 3.4 & $3 \cdot 2$ & 3.4 & $3 \cdot 2$ & $3 \cdot 2$ & 3.2 & 3.9 & $2 \cdot 8$ & $4 \cdot 0$ & 3.2 & 3.5 & $2 \cdot 5 \S$ \\
\hline Energy (KJ (kcal/d)) & 9878 (2361) & 7694 (1839) & 6878 (1644) & 6899 (1649)† & 7251 (1733) & $7364(1760)$ & 7632 (1824) & $9113(2178) \dagger$ & 7058 (1687) & 7312 (1748) & 7485 (1789) & 9498 (2270)‡ \\
\hline $\begin{array}{l}\text { Age on questionnaire } \\
\text { completion (years) }\end{array}$ & $42 \cdot 6$ & 43.7 & $42 \cdot 0$ & 41.5 & $46 \cdot 0$ & 44.6 & 41.5 & $37.5 \dagger$ & 39.9 & $40 \cdot 9$ & 44.4 & $44.5 \ddagger$ \\
\hline Weight now (kg) & $92 \cdot 3$ & 93.6 & 89.7 & $95 \cdot 7$ & $88 \cdot 1$ & $92 \cdot 3$ & $94 \cdot 2$ & $96 \cdot 8 \ddagger$ & 93.5 & 93.8 & 93.4 & $90 \cdot 7$ \\
\hline BMI now $\left(\mathrm{kg} / \mathrm{m}^{2}\right)$ & 33.9 & 33.7 & 33.0 & $35 \cdot 0$ & $32 \cdot 3$ & 33.9 & $34 \cdot 3$ & $35 \cdot 2 \ddagger$ & $34 \cdot 3$ & 34.5 & $34 \cdot 1$ & $32 \cdot 9$ \\
\hline $\begin{array}{l}\text { Percentage weight } \\
\text { loss (\%) }\end{array}$ & $-2 \cdot 9$ & $-3 \cdot 4$ & $-3 \cdot 4$ & $-3 \cdot 3$ & $-4 \cdot 1$ & $-2 \cdot 8$ & $-4 \cdot 0$ & $-2 \cdot 2 \ddagger$ & $-3 \cdot 8$ & $-3 \cdot 1$ & $-3 \cdot 4$ & $-2 \cdot 8$ \\
\hline Current smokers (\%) & 7.5 & 4.9 & $6 \cdot 0$ & $13 \cdot 9$ & 11.3 & 8.5 & 7.5 & 7.5 & 11.4 & 9.9 & $6 \cdot 2$ & 7.4 \\
\hline $\begin{array}{l}\text { Exercise (frequency/ } \\
\text { week) }\end{array}$ & 8.9 & $9 \cdot 0$ & $7 \cdot 6$ & $8 \cdot 7$ & 9.5 & $8 \cdot 3$ & $8 \cdot 6$ & $7 \cdot 8$ & $7 \cdot 3$ & $8 \cdot 6$ & $9 \cdot 1$ & $9 \cdot 1 \ddagger$ \\
\hline Employed (\%) & $74 \cdot 1$ & $79 \cdot 3$ & $77 \cdot 2$ & 77.5 & $78 \cdot 8$ & 69.5 & $75 \cdot 0$ & $85 \cdot 0$ & 77.5 & $74 \cdot 1$ & $81 \cdot 3$ & $75 \cdot 3$ \\
\hline $\begin{array}{l}\text { Vitamin and mineral } \\
\text { supplement use (\%) }\end{array}$ & $40 \cdot 5$ & $45 \cdot 1$ & 35.4 & $31 \cdot 3$ & $38 \cdot 8$ & 34.6 & 33.3 & $46 \cdot 2$ & $36 \cdot 3$ & 35.4 & $36 \cdot 3$ & 44.4 \\
\hline \multicolumn{13}{|l|}{ Breakfast (\%) } \\
\hline Always & $80 \cdot 2$ & 87.8 & $95 \cdot 1$ & $77 \cdot 1$ & 91.4 & 89.0 & $82 \cdot 1$ & $74.7 \|$ & $82 \cdot 7$ & 84.8 & $87 \cdot 8$ & $90 \cdot 1$ \\
\hline Sometimes & 9.9 & $7 \cdot 3$ & 2.5 & $13 \cdot 6$ & $6 \cdot 2$ & 3.7 & $12 \cdot 6$ & $13 \cdot 3$ & $9 \cdot 9$ & $10 \cdot 1$ & $9 \cdot 8$ & $3 \cdot 7$ \\
\hline Never & 9.9 & 4.9 & 2.5 & 3.7 & 2.5 & $7 \cdot 3$ & 4.8 & $12 \cdot 6$ & $7 \cdot 4$ & $5 \cdot 1$ & 2.4 & $6 \cdot 2$ \\
\hline Fibre $(g / d)$ & $40 \cdot 8$ & $32 \cdot 1$ & $29 \cdot 3$ & $27 \cdot 5 \dagger$ & 33.5 & 32.5 & 31.4 & $32 \cdot 4$ & $30 \cdot 3$ & 30.4 & $31 \cdot 2$ & $37 \cdot 8$ \\
\hline $\begin{array}{l}\text { Free sugar } \\
\qquad \% \text { total kcal) }\end{array}$ & 9.5 & 8.7 & 8.2 & $8.4 \ddagger$ & $8 \cdot 3$ & $7 \cdot 7$ & 9.1 & $9.9 \dagger$ & 8.9 & 8.5 & $8 \cdot 8$ & 8.7 \\
\hline
\end{tabular}

HFSS, high fat, salt and sugar; $Q$, quartile.

${ }^{*} P$-values $<0.05$ were considered as significant. Exercise expressed as total frequency of vigorous and moderate physical activity and walking events lasting for at least 10 min/event per week.

$\dagger P$ for trend $<0.001$ calculated using Jonckheere-Terpstra test.

$\ddagger P$ for trend $0.001<P<0.025$ calculated using Jonckheere-Terpstra test.

$\S P$ for trend $0.025<P<0.05$ calculated using Jonckheere-Terpstra test.

$\|$ Chi square test of independence of factor quartile against category of breakfast consumption, $P<0.025$, for all categories of breakfast consumption

Otherwise no significant $P$ for trend. 
Quartile (Q) of baseline factor score

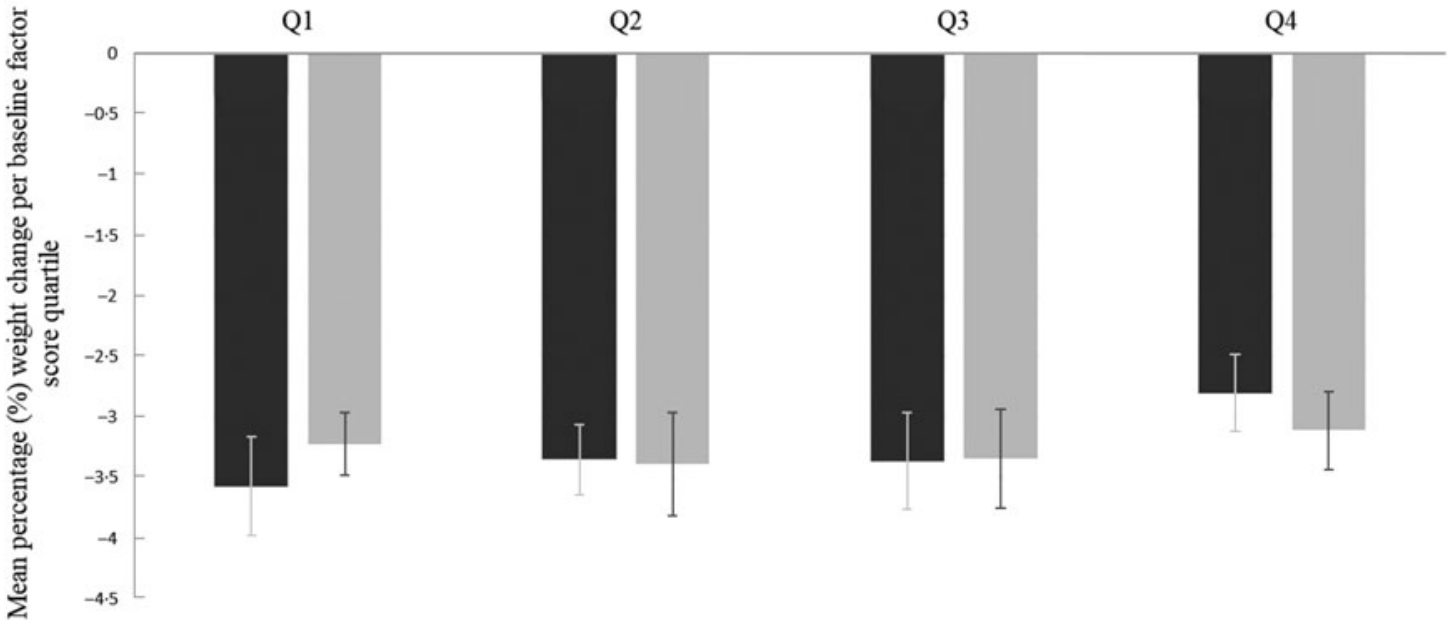

Fig. 2 Percentage weight change per quartile of factor score at baseline. Data are presented as a mean \pm SE of the mean. $P$ for trend of 'plant based' $(\square)=0.046$. P for trend for 'processed meat and sugar added by the consumer' $(\square)=0.249$. Significance of $P$ for trend was set at $P<0.05$. Significance was calculated using the Jonckheere-Terpstra test

\section{Discussion}

Previously, the extent to which commercial weight management programmes promote adherence to SACN's dietary fibre and free sugar recommendations has been unknown. This research identified that a greater number of participants met fibre and free sugar recommendations through engagement in a weight management programme, related to significant increased consumption of fresh fruit, vegetables, legumes and whole grains alongside a decreased intake of processed meat and confectionery.

A high 'plant based' DP score prior to joining SW was associated with a lower percentage body weight loss with engagement. A suggested mechanism is that a 'plant based' DP closely aligns with SW's Food Optimising plan and Eatwell guide; thus, individuals may have more difficulty in reducing energy intake than those with poorer $\mathrm{DP}^{(3,8)}$. A secondary analysis of overweight individuals taking part in a 3-month clinical weight-loss trial reported similar results $^{(9)}$. This suggests that initial DP may predict weight loss with engagement ${ }^{(8)}$.

A low 'HFSS products' DP score at T1 correlated with greater percentage body weight loss. In a separate study, those who lost a clinically significant $\geq 5 \%$ body weight consumed significantly less energy dense nutrient poor (HFSS) foods, compared with those who lost $<5 \%$ body weight $^{(28)}$. A plausible mechanism is that energy-dense, nutrient-poor foods are less satiating, leading to greater hunger experiences and overeating ${ }^{(29)}$. A low 'HFSS products' DP was also associated with lower free sugar consumption, lower energy intake, having breakfast every day and a mean fibre intake that meets SACN's recommendation.

Before engagement, the DP identified could be described as either 'more healthy' or 'less healthy' (plant based $v$. processed meat and sugar added by the consumer). This indicates an underlying dichotomous thinking style at T0 may be present, in which individuals follow an entirely healthy diet or not bother at all ${ }^{(30)}$. After joining $\mathrm{SW}$, a different set of three DP emerged. This change may result from participants following the core SW diet of 'free foods', and the DP indicates how participants use their allowance of 'healthy extras' and 'syns' ${ }^{\text {(8) }}$. Length of membership negatively correlated with a 'nuts and oils' pattern, suggesting participants altered their diet to include less of the above energy-dense items as length of membership increased. This may be through members choosing to use their allowance of 'healthy extras', in which nuts are included, on cereals or bread (significant increased consumption with engagement as a percentage of energy intake, Fig. 1) and choosing HFSS products as 'syns' rather than additional servings of nuts and oils.

The changes made by the participants reflect changes seen in a previously published analysis of individuals participating in a clinical weight-loss trial. Significant increases in vegetable, legume, whole grain and fruit consumption were seen, whilst significant decreases in participants processed and fatty meats, nuts and seeds and HFSS product consumption were reported, measured through diet histories taken at baseline and 3 months ${ }^{(31)}$.

Through engagement in SW, mean dietary fibre intake increased, partly related to SW participants buying more fruit and vegetables, which are typically high in fibre ${ }^{(32,33)}$. The National Diet and Nutrition Survey reported in 2014/ $2015-2015 / 2016$ the mean dietary fibre intake in women 19-64 years of age was $17.4 \mathrm{~g} / \mathrm{d}^{(34)}$. It could indicate this group consumed more fibre than the UK population average. However, overweight individuals tend to over report intakes of fruit and vegetables ${ }^{(35)}$ and actual portion sizes may differ from portion sizes taken from the FFQ. 
At T0, a significant positive correlation between free sugar consumption as a percentage of total dietary energy and total energy intake was seen. This is in line with SACN's report that free sugar consumption is linked to a greater energy intake ${ }^{(2)}$. At T1, there was no significant correlation between free sugar and energy intake or free sugar and weight loss. This may result from Food Optimising. Food Optimising promotes dietary changes which impact on a wide range of nutrients to induce a negative energy balance, such that free sugar intake alone may not predict weight loss.

The analysis has many strengths. An adequate sample size for PCA to return reliable and accurate DP was used. By energy adjusting the food groups, direct comparison between food group intakes as a proportion of the diet at the two time points was permitted. The data are collected from a community-based sample of people trying to lose weight, thus increasing the ecological validity of the results.

As limitations, the current study investigated the initial, short-term, changes in DP with engagement in a weight management programme; therefore, it remains unknown if the changes in DP are maintained over a longer period. The length of membership varied amongst participants, despite this, length of membership only significantly correlated with a decreased agreement with a 'nuts and oil' DP. As a retrospective design was used to collect dietary intake at T0, the reliability could be affected by recall bias, new education provided through engagement with SW and no reference to a timeframe for participants to report their retrospective intake. FFQ limit participants to report only their intake of the food items included within the questionnaire, it is noted that some sources of free sugars were not covered by the FFQ. The FFQ used in this analysis was validated against a 4 -d food record at two separate time points rather than simultaneously. The FFQ used was validated using a New Zealand population, at the time of conducting the research no such FFQ validated in a UK population for the use of identifying DP, dietary fibre and free sugar intake existed. The retained DP accounted for 17.6 and $21.0 \%$ of the variance present in the dataset at T0 and T1, respectively. Whilst conceivably low, this is in the range of previous reports ${ }^{(10,15,22)}$. At present, there are limited published reports of the free sugar content of foods; thus, this analysis used an Australian database detailing the content of sugar added to foods via the manufacturer. The FFQ did not collect data on the participants' ethnicity. A major limitation is the crude estimation of physical activity, which did not take into account the intensity of physical activity.

\section{Conclusions for practice}

Through the SW programme, a significant increase in the proportion of participants meeting the SACN recommendations was seen. Despite this, the numbers meeting the recommendations represented a small proportion of participants. This indicates further work is required for the maximum of $5 \%$ energy derived from free sugar and the dietary reference value for dietary fibre to be achieved by all members. The research suggests that DP prior to engagement in a weight management programme have some ability to predict individuals' initial weight loss. This research provides further evidence to support the promotion of a diet low in HFSS products to individuals wishing to lose weight and lower energy and free sugar intake. Healthcare professionals can use the results to tailor their signposting of patients to weight management services and to aid the translation of nutrient-based recommendations into practical advice for their patients. Future research needs to determine the DP in a male and female population engaging in a commercial weight management programme, over a longer period.

\section{Acknowledgements}

Acknowledgements: We are very grateful to those SW members who kindly participated in this research and Slimming World for allowing access to their members through placing the Fe FFQ on their website. Financial support: This research received no specific grant from any funding agency, commercial or not-for-profit sectors. Conflict of interest: We need to declare that A.A., alongside her academic position at the University of Nottingham, also holds a consultancy position at Slimming World. The survey was hosted by Slimming World. Authorship: L.S. was responsible for designing the study, carrying out the secondary analysis and drafted the article, H.K.K was responsible for collecting the primary FFQ data, A.A. supervised the research and formulated the research questions. Ethics of human subject participation: The current study was conducted according to the guidelines laid down in the Declaration of Helsinki, and all procedures involving research study participants were approved by the School of Biosciences Research Ethics Committee at the University of Nottingham (ethics number: SBREC150132A). Written informed consent was obtained from all subjects.

\section{Supplementary material}

For supplementary material accompanying this paper visit https://doi.org/10.1017/S1368980019004762

\section{References}

1. NHS Digital (2017) Statistics on Obesity, Physical Activity and Diet. National Statistics. https://www.gov.uk/government/ statistics/statistics-on-obesity-physical-activity-and-dietengland-2017 (accessed December 2017). 
2. Scientific Advisory Committee on Nutrition (2015) Carbohydrates and Health. London, UK. https://assets.publishing. service.gov.uk/government/uploads/system/uploads/ attachment_data/file/445503/SACN_Carbohydrates_and_ Health.pdf (accessed January 2019).

3. Cobiac LJ, Scarborough P, Kaur A et al. (2016) The Eatwell guide: modelling the health implications of incorporating new sugar and fibre guidelines. PLOS ONE 11, 1-16.

4. Truby H (2006) Randomised controlled trial of four commercial weight loss programmes in the UK: initial findings from the BBC "diet trials". Br Med J 332, 1309-1314.

5. Jolly K, Daley A, Adab P et al. (2010) A randomised controlled trial to compare a range of commercial or primary care led weight reduction programmes with a minimal intervention control for weight loss in obesity: the lighten up trial. BMC Public Health 10, 439.

6. Stubbs RJ, Morris L, Pallister C et al. (2015) Weight outcomes audit in 1.3 million adults during their first 3 months' attendance in a commercial weight management programme. BMC Public Health 15, 882.

7. National Institute for Health and Care Excellence (2017) Obesity: identification, assessment and management [CG189]. National Institute for Health and Care Excellence. https://www.nice.org.uk/guidance/cg189/ifp/chapter/obesityand-being-overweight (accessed January 2019).

8. Slimming World (2017) Dietary approach - food optimising https://www.slimmingworld.co.uk/health/how-sw-works/ food-optimising.aspx (accessed January 2019).

9. Grafenauer SJ, Tapsell LC, Beck EJ et al. (2013) Baseline dietary patterns are a significant consideration in correcting dietary exposure for weight loss. EurJ Clin Nutr 67, 330-336.

10. Luger E, Aspalter R, Luger M et al. (2016) Changes of dietary patterns during participation in a web-based weightreduction programme. Public Health Nutr 19, 1211-1221.

11. Huseinovic E, Winkvist A, Bertz F et al. (2014) Changes in food choice during a successful weight loss trial in overweight and obese postpartum women. Obesity 22, 2517-2523.

12. Hall KD, Sacks G, Chandramohan DL et al. (2011) Quantification of the effect of energy imbalance on bodyweight. Lancet 378, 826-837.

13. Tang J, Abraham C, Greaves C et al. (2014) Self-directed interventions to promote weight loss: a systematic review of reviews. J Med Internet Res 16, 584 .

14. Beck KL \& Heath ALM (2013) Dietary approaches to assessing iron-related nutrition. Curr Opin Clin Nutr Metab Care 16, 712-718.

15. Beck KL, Kruger R, Conlon CA et al. (2012) The relative validity and reproducibility of an iron food frequency questionnaire for identifying iron-related dietary patterns in young women. J Acad Nutr Diet 112, 1177-1187.

16. Finglas PM, Roe MA, Pinchen HM et al. (2015) McCance and Widdowson's the Composition of Foods, 7th ed. Cambridge: The Royal Society of Chemistry.

17. Nutritics (2017) Nutritics Nutritional Analysis Software. Nutritics Education v4.315.

18. Swan GE, Powell NA, Knowles BL et al. (2018) A definition of free sugars for the UK. Public Health Nutr 21, 1636-1638.
19. Louie JCY, Moshtaghian H, Boylan S et al. (2015) A systematic methodology to estimate added sugar content of foods. Eur J Clin Nutr 69, 154-161.

20. Ambrosini GL, O'Sullivan TA, de Klerk NH et al. (2011) Relative validity of adolescent dietary patterns: comparison of a food frequency questionnaire and 3-day food record. Br J Nutr 105, 625-633.

21. Calvert C, Cade J, Barrett JH et al. (1997) Using cross-check questions to address the problem of mis-reporting of specific food groups on food frequency questionnaires. Eur J Clin Nutr 51, 708-712.

22. Hu FB (2002) Dietary pattern analysis: a new direction in nutritional epidemiology. Curr Opin Lipidol 13, 3-9.

23. Flood A, Rastogi T, Wirfalt E et al. (2008) Dietary patterns as identified by factor analysis and colorectal cancer among middle-aged Americans. Am J Clin Nutr 88, 176-184.

24. O'Rourke N \& Hatcher L (2014) A Step-by-Step Approach to Using SAS for Factor Analysis and Structural Equation Modeling, 2nd ed. Cary, North Carolina: SAS Institute Inc.

25. Reedy J, Wirfalt E, Flood A et al. (2010) Comparing 3 dietary pattern methods-cluster analysis, factor analysis, and index analysis-with colorectal cancer risk. Am J Epidemiol 171 479-487.

26. Field A (2005) Discovering Statistics Using SPSS, 2nd ed. London: Sage Publications Ltd.

27. IBM Corp (2013) IBM SPSS Statistics for Windows. Version 22.0. Armonk, NY: IBM Corp.

28. Hutchesson MJ, Collins CE, Morgan PJ, et al. (2014) Changes to dietary intake during a 12 -week commercial web-based weight loss program: a randomized controlled trial. Eur $J$ Clin Nutr 68, 64-70.

29. Fuhrman J, Sarter B, Glaser D et al. (2010) Changing perceptions of hunger on a high nutrient density diet. Nutr $\mathbf{9} \mathbf{9}, 51$.

30. Byrne S, Cooper Z \& Fairburn C (2003) Weight maintenance and relapse in obesity: a qualitative study. Int J Obes $\mathbf{2 7}$, 955-962.

31. Grafenauer SJ, Tapsell LC, Beck EJ et al. (2015) Changes in food choice patterns in a weight loss intervention. Nutr Diet 72, 309-315.

32. Pallister C, Avery A, Stubbs J et al. (2009) Influence of slimming world's lifestyle programme on diet, activity behaviour and health of participants and their families.J Hum Nutr Dietet 22, 351-358.

33. National Research Council (US) \& Committee on Diet and Health (1989) Diet and Health: Implications for Reducing Chronic Disease Risk, 1st ed. Washington (DC): National Academies Press.

34. Roberts C, Steer T, Maplethorpe N et al. (2018) National Diet and Nutrition Survey. Results from Years 7 and 8 (combined) of the Rolling Programme (2014/2015 To 2015/2016). London: Public Health England. https://assets. publishing.service.gov.uk/government/uploads/system/ uploads/attachment_data/file/699241/NDNS_results_years_7_ and_8.pdf (accessed January 2019).

35. Macdiarmid J \& Blundell J (1998) Assessing dietary intake: who, what and why of under-reporting. Nutr Res Rev 11, 231-253. 